\title{
Das Bildungssystem der Türkei. Schulische Bildungslandschaft, nationale, internationale und zivilgesellschaftliche Einflussfaktoren
}

\author{
Inga Schwarz
}

\section{$1 \quad$ Einleitung}

In Bezug auf das türkische Bildungswesen lässt sich sowohl im Hinblick auf Überblicksliteratur als auch auf aktuelle Debatten eine Forschungslücke in der deutschsprachigen und in der internationalen Wissenschaft feststellen. Nohl et al. merken daher zu Recht an, »[1] ooking at the international scientific discourse, it seems there is surprisingly little work on the Turkish education system «1. Diese Forschungslücke verwundert, müssen dem türkischen Bildungssystem doch eklatante Defizite konstatiert werden. Im internationalen Bildungswettbewerb schneidet die Türkei bei Untersuchungen der Organization for Economic Co-operation and Development (OECD) und im Programme for International Student Assessment (PISA) unterdurchschnittlich ab. Mit Blick auf eine globalisierte, wissenschaftsgestützte Ökonomie, die eine international vergleichbare und hochwertige Bildung verlangt, scheint ein grundlegender Wandel im Bereich des Bildungssystems auch für das türkische Bildungssystem unumgänglich. Seit den 1990er Jahren fordert etwa die Vereinigung türkischer Industrieller und Geschäftsleute (Türk Sanayicileri ve İşadamları Derneği, TÜSİAD) die Neustrukturierung des Bildungssystems, das bisher junge Menschen hervorbringe, welche »Hochtechnologien gegenüber unsensibel sind, den Ansprüchen der Informationsgesellschaft nicht genügen, keine einzige Fremdsprache sprechen, sich den Stoff nur einpauken und

1 Nohl 2008, S. 7. Nohl et al. selbst wirken dieser Erscheinung mit ihrem in der Reihe »European Studies in Education « bei Waxmann 2008 veröffentlichten Band »Education in Turkey» entgegen, indem sie eine fundierte Einführung in das türkische Bildungssystem und aktuelle Debatten vorlegen, die sie zum Teil aus dem Türkischen übersetzen. Im Jahr 2011 folgte mit »Bildung und sozialer Wandel in der Türkei - Historische und aktuelle Aspekte « eine weitere Publikation Nohls, in der ebenfalls auf aktuelle Debatten um das Bildungssystem der Türkei eingegangen wird. 
eindimensional, unflexibel, dogmatisch und lehrerzentriert funktionieren. $\aleph^{2}$ Die amtierende Regierungspartei AKP (Adalet ve Kalkınma Partisi, Partei für Gerechtigkeit und Entwicklung) schloss sich dieser Meinung an und erklärte nicht nur, dass das türkische Bildungssystem den Ansprüchen des 21. Jahrhunderts in wirtschaftlicher Hinsicht nicht gewachsen sei, sondern auch, dass sie eine umfassende Neustrukturierung desselben durchführen werde. Besonders in Hinblick auf die hohe Arbeitslosigkeitsrate sieht sich Ministerpräsident Erdoğan zu Reformen gezwungen. »As Turkey strives to keep its economy forging ahead despite persistent global economic and financial crises, Erdoğan has placed a high priority on sustaining the links between better education, stronger human capital, and higher rates of employment. « ${ }^{3}$

Der vorliegende Artikel gibt Einblick in das Bildungssystem der Türkei und dessen Reformbedürftigkeit und zeigt Bestrebungen zur Neustrukturierung der Bildungslandschaft seitens staatlicher, internationaler und zivilgesellschaftlichen Akteure auf. Zu diesem Zweck wird zunächst eine Einführung in das türkische Bildungssystem und dessen aktuelle Problemlagen gegeben. Im zweiten Teil wird auf die Spannung zwischen verschiedenen Einflussfaktoren auf das Bildungswesen hingewiesen, die auf nationaler und internationaler Ebene bestehen. Verdeutlicht werden diese Spannungen an der Bildungsreform von 2012. In einem dritten Teil wird schließlich auf die Umsetzung diffundierender Bildungsstrategien durch zivilgesellschaftliche Akteure eingegangen, die ebenfalls einen Beitrag zur Neustrukturierung des türkischen Bildungssystems leisten.

\section{Das Bildungssystem der Republik der Türkei}

In der Betrachtung des türkischen Bildungswesens lassen sich zunächst zwei Bereiche, das formale und das non-formale Bildungssystem, unterscheiden. Das in dieser Studie analysierte formale Bildungssystem beinhaltet Vor-, Primar-, Sekundar- und Hochschulbildung, während sich der non-formale Bildungssektor außerhalb des regulären Schulwesens auf weiterbildende Maßnahmen der Erwachsenenbildung bezieht. Beide Teilbereiche des türkischen Bildungswesens stehen unter der Aufsicht des Ministeriums für Nationale Bildung (Milli Ĕ̆itim Bakanlı̆gl, MEB), das bereits seit der Entstehung des türkischen Bildungssystems unter verschiedenen Bezeichnungen und in verschiedener Position Einfluss auf die Ausbildung türkischer Bürger ausübt.

2 İnal 2011, S. 62.

3 İlhan 2012, S. 92. 


\section{Abbildung 1}

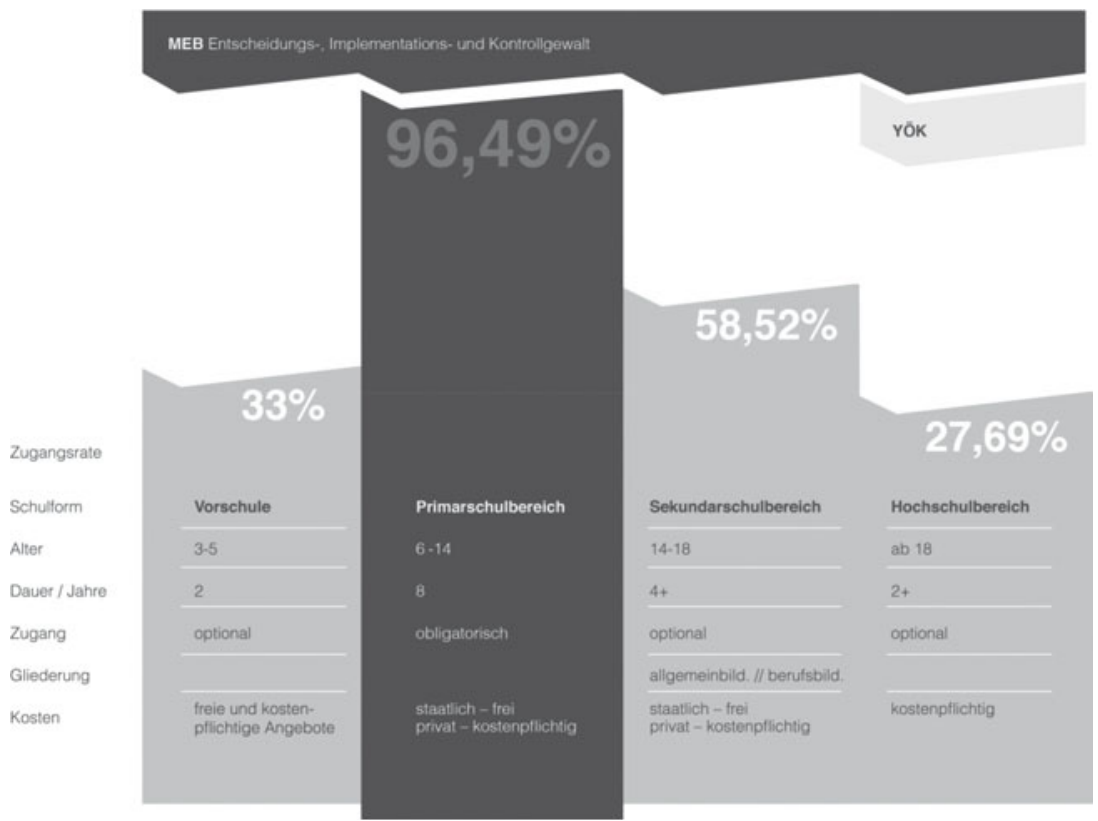

Quelle: Eigene Grafik - Statistische Daten aus dem Schuljahr 2008/09, entnommen aus Ministry of National Education $2009 *$ Zugangsrate zur Vorschule 2008/09 aus Ministry of National Education 2008, S. 4.

Aus der oben stehenden Grafik lässt sich ein Überblick über Organisationsstruktur, Schulform, Altersgruppen und Zugangsrate zu den jeweiligen Institutionen gewinnen, deren Beschaffenheit im Folgenden näher erläutert wird. Die aktuelle Organisation des türkischen Bildungswesens wird in naher Zukunft umfassenden Reformen unterworfen werden, auf die in Abschnitt 3 eingangen wird.

\subsection{Das Ministerium für Nationale Bildung als oberste Lenkungs- und Kontrollbehörde}

Das osmanische Ministerium für Nationale Bildung wurde 1857 gegründet und trägt seinen heutigen Namen seit 1992. Das Ministerium ist bis heute das oberste Entwicklungs- und Kontrollorgan des nationalen Bildungsprogramms. Dabei ist es dem kemalistischen Nationalgedanken gesetzlich verpflichtet, wie das 1992 er- 
lassene Gesetz Nr. 3797 vorgibt, das die Zuständigkeit des Ministeriums wie folgt zusammenfasst:

»to plan, program, implement, monitor and control education and training services targeted at teachers and students in the educational institutions at all levels affiliated to the Ministry with the objective of raising individuals who are commited to Atatürk's principles and reforms, and to the [sic!] Atatürk's nationalism defined in the Constitution of the Republic of Turkey, who adopt, protect and develop the national, ethical, spiritual, historical and cultural values of the Turkish nation, who love and elevate their families, homeland and nation, who are aware of their duties and responsibilities to the Republic of Turkey - which is a democratic, secular and social state ruled by law and based on human rights and the basic principles defined at the beginning of the Constitution - and who behave accordingly. « ${ }^{4}$

$\mathrm{Zu}$ den Aufgabenbereichen des Ministeriums gehören die Schaffung bzw. Autorisierung aller formalen und non-formalen Bildungseinrichtungen außerhalb der Hochschulbildung. Es organisiert die Bildung türkischer Staatsbürger im In- und Ausland, legt die Lehrpläne und Abschlüsse aller Bildungseinrichtungen außer der Hochschule fest, regelt die Koordination der an die türkische Armee angegliederten Bildungseinrichtungen, die Kontrolle der Einhaltung nationaler Richtlinien im Hochschulbetrieb, die Umsetzung von physischer Bildung in schulischen Einrichtungen sowie die Bereitstellung von Unterbringung und finanzieller Unterstützung von Studierenden. ${ }^{5}$ Das Ministerium beeinflusst aufgrund dieses umfangreichen Zuständigkeitsbereiches die im Folgenden dargestellten Schulformen gleichermaßen und sieht sich nur in Bezug auf das Hochschulwesen Einschränkungen ausgesetzt.

\subsection{Vorschulische Bildungseinrichtungen}

Frühkindliche Erziehung wird von staatlichen und privaten Einrichtungen angeboten und gehört zu den optionalen Bildungsangeboten. Die Einrichtungen können als unabhängiger Kindergarten oder angegliedert an Grundschulen eröffnet werden und bieten Vorbereitung auf die Grundschulausbildung für die Altersgruppe der Drei- bis Fünfjährigen. Ihr Angebot folgt dem Lehrplan der Generaldirektion für Vorschulen des Ministeriums für Nationale Bildung. ${ }^{6}$ Ungenü-

4 Ministry of National Education 2005, S. 4 f.

5 Vgl. ebd., S. 5.

6 Vgl. Ministry of National Education 2009, S. XI. 
gendes Angebot, das vor allem in ländlichen Regionen im Südosten des Landes zu verzeichnen ist, führt zu niedrigen Zugangsraten und lässt die OECD in ihrem Bildungsbericht zur Türkei aus dem Jahr 2007 zu der Einschätzung kommen, »that the general situation in pre-primary education [...] requires considerable improvement. $\ll^{7}$ Diese Einschätzung bestätigt sich im Blick auf die Zugangschancen zu vorschulischer Bildung. Mit einer Zugangsrate von 33 Prozent im Schuljahr 2008/o9 liegt die Türkei weit hinter dem Durchschnittswert der OECD von 67,8 Prozent und beispielsweise den Werten der Bundesrepublik Deutschland zurück. ${ }^{8}$

Die Zugangschancen lassen ein starkes Gefälle zwischen urbanen und ruralen Gebieten erkennen. So lag die Zugangsrate 2007 für die ägäische Region bei 22 Prozent, während in Ostanatolien nur neun Prozent der entsprechenden Altersgruppe vorschulische Bildungseinrichtungen nutzen konnten. ${ }^{9}$ Besonders niedrige Zugangsraten bestehen in der vorschulischen Bildung für Mädchen, die lediglich 48 Prozent der bereits niedrigen Zugangsrate stellen. ${ }^{10}$ Ausnahmen von der niedrigen Zugangsrate bilden hingegen reiche Distrikte in Istanbul, in denen 50 Prozent der Drei- bis Fünfjährigen eine frühkindliche Erziehung genießen. Diese hohe Zugangsrate weist auf eine weitere Ungleichheit im vorschulischen Bildungsbereich hin, die Kindern aus wirtschaftlich benachteiligten Familien den Zugang zu Bildungseinrichtungen zusätzlich erschwert. So finden sich in den wohlhabenden Stadtbezirken Istanbuls mit hoher frühkindlicher Bildungsrate zahlreiche private Kindergärten, die bessere Betreuungskonditionen anbieten, deren monatliche Gebühr jedoch nur von wohlhabenden Eltern geleistet werden kann. ${ }^{11}$

Mit der Einführung von an Grundschulen angegliederten, kostenfreien einjährigen vorschulischen Angeboten konnte die Zugangsrate zu frühkindlicher Erziehung in den letzten Jahren beträchtlich angehoben werden. Lagen die $\mathrm{Zu}-$ gangsraten 2003 noch bei elf Prozent, so konnten diese bis 2008/09 auf 33 Prozent angehoben werden. ${ }^{12}$ Damit wurde bereits eine Verbesserung der Zugangs-

7 Vgl. OECD 2007, S. 34 .

8 Vgl. Ministry of National Education 2008, S. 4 bzw. OECD 2007, S. 35. In Deutschland ist die Bildungsbeteiligung der Vier- und Fünfjährigen ebenfalls optional und hält sich trotz dessen bundesweit seit 2008 bei über 95 Prozent. Vgl. Autorengruppe Bildungsberichterstattung 2010, S. 49.

9 OECD 2007, S. 35; Ministry of National Education 2009, S. 47-52.

10 Ministry of National Education 2009, S. 47.

11 So werden 2011 für den einkommensniedrigen Stadtbezirk Fatih mit 429 ooo Einwohnern 2700 Registrierungen für vorschulische Einrichtungen angegeben, während der reiche Distrikt Sarryer mit nur 287000 Einwohnern 3500 Registrierungen verzeichnen kann. Vgl. İstanbul İl Milli Eğitim Müdürlügü 2012, S. 40.

12 Vgl. Ministry of National Education 2008, S. 4. 
chancen für sozio-ökonomisch benachteiligte Familien eingeleitet. Ob jedoch die durch das Ministerium für Nationale Bildung festgelegte Zielsetzung einer flächendeckenden 50-prozentigen Zugangsrate bis zum Jahr $2013^{13}$ erreicht werden kann, bleibt abzuwarten. Mit Blick auf die Statistiken des Schuljahres 2010/11 jedoch stellt sich ein solch eklatanter Anstieg der Zugangsrate als unwahrscheinlich heraus, stagniert die Rate seit 2008/09 doch bei 33 Prozent. ${ }^{14}$

Die mit dem Zugang zu frühkindlicher Bildung proportional einhergehende Kindersterblichkeitsrate, die 2010 in der Türkei bei unter Fünfjährigen mit 18 Todesfällen pro 1000 Lebendgeburten im Vergleich zu vier Todesfällen in Deutschland extrem hoch lag, ${ }^{15}$ hat den Bereich der frühkindlichen Bildung in der Republik Türkei verstärkt in den Blick internationaler Initiativen rücken lassen. ${ }^{16}$ So beteiligt sich UNICEF in Kooperation mit der Europäischen Union und der Weltbank intensiv an Kampagnen zur Umsetzung erhöhter Bildungsraten im Bereich der frühkindlichen Erziehung und zur Förderung der höheren Beteiligungsrate von Mädchen am schulischen Betrieb. Der OECD-Bericht stellte 2007 zu Recht fest, dass "Turkey's ECEC [early childhood education and care, eigene Anmerkung] policies and programs appear to be driven primarily by international organizations and not by a formal commitment of the government $[\ldots] . \ll^{17}$ Eine umfassende Kampagne der türkischen Regierung in diesem Bereich steht bisher noch aus.

\subsection{Primarschulwesen}

Seit 1997 besteht in der Republik der Türkei eine achtjährige Grundschulpflicht. Diese bildet bisher die einzig obligatorische Grundlage des türkischen Bildungssystems und wird an staatlichen Schulen kostenfrei angeboten. ${ }^{18}$ Gemäß dem ersten Artikel des Gesetzes zur Primarschulbildung (222/1961) ist diese "the basic schooling that serves all Turks of both sexes and their physical, mental and mo-

13 Vgl. ebd., S. 12.

14 Der Bericht gibt in Bezugnahme auf das Schuljahr 2009/10 für die Altersstufe der Drei- bis Fünfjährigen eine Zugangsrate von 27 und für die Vier- bis Fünfjährigen 38,6 Prozent an. Daraus ergibt sich ein Mittel von 32,8 Prozent. Vgl. Ministry of National Education 2011, S. 2.

15 Vgl. UNICEF 2010.

16 Der Zusammenhang zwischen frühkindlicher Bildung und Kindersterblichkeitsrate besteht über der gesicherten medizinischen Versorgung für Kleinkinder und der Aufklärung der Eltern über gesundheitliche Risiken.

17 OECD 2007, S 39.

18 Zur geplanten Bildungsreform, durch die die Schulpflicht auf 12 Jahre festgesetzt wird, vgl. Abschnitt 3. 
ral development in accordance with national aims ${ }^{19}$. Registrierungs- und Beteiligungsraten konnten aufgrund der oben genannten, mit dem Gesetz von 1997 einhergehenden Reformen des Grundschulwesens signifikant angehoben werden. Die umfassenden Reformen waren aufgrund wirtschaftlicher Notwendigkeiten umgesetzt worden und zielten darauf ab, besonders Kindern aus sozioökonomisch benachteiligten Familien den Zugang zur Schulbildung zu erleichtern. Die verpflichtende Schuldauer wurde im Zuge dessen von fünf auf acht Jahre angehoben, Lehrinhalte internationalen Standards angepasst sowie vorhandene schulische Einrichtungen aus- und neue Schulen aufgebaut. ${ }^{20}$ Die Zugangsrate, die noch 1997/98 unter 90 Prozent lag, erreichte daraufhin bis zum Schuljahr 2009/10 98,2 Prozent der entsprechenden Altersgruppe. ${ }^{21}$ Bis zum Jahr 2013 hat sich das Ministerium für Nationale Bildung zum Ziel gesetzt, eine hundertprozentige Schulrate für Sechs- bis 14-Jährige zu erreichen. ${ }^{22} \mathrm{Um}$ dies zu realisieren müssten jedoch die im Folgenden zu erörternden aktuellen Probleme des türkischen Primarschulwesens behoben werden.

\section{Bildungsgefälle zwischen westlich-urbanen und östlich-ruralen Gebieten}

Bereits in der Entstehungsphase der Republik der Türkei setzte sich die Regierung das Ziel, der Landbevölkerung, die zu dieser Zeit 76 Prozent der Bevölkerung umfasste und nahezu ausschließlich aus Analphabeten und Analphabetinnen bestand, die Grundschulausbildung zugänglich zu machen. ${ }^{23}$ Heute leben zwar nur noch 29 Prozent der türkischen Bevölkerung in ländlichen Gebieten, ein erschwerter Zugang zur Grundschulausbildung besteht jedoch bis heute fort. Vor allem ländliche Gebiete im Südosten sind noch weit von der für 2013 angestrebten 100 Prozentrate der Einschulungsquote entfernt. So lässt sich in der Bildungsstatistik zum Schuljahr 2008/o9 eine Aufschlüsselung der Zugangsrate zur Primarschulbildung von 96,5 Prozent finden, die den Westmetropolen Istanbul und Izmir eine Zugangsrate von 98,6 bzw. 99,2 Prozent bescheinigt. Die Provinzen Van und Muş im Südosten mit hohem Anteil an ländlichen Gebieten hingegen, kommen lediglich auf eine Zugangsrate von 88,2 bzw. 87,7 Prozent zur obligatorischen Primarschulbildung. ${ }^{24}$ Maßnahmen zur besseren Anbindung ländlicher

19 Zitiert nach Gümüş 2008, S. 49.

20 Ausführlicher zur Bildungsreform von 1997 vgl. etwa Dulger 2004.

21 Vgl. Ministry of National Education 2011, S. 2.

22 Vgl. Ministry of National Education 2008, S. 12.

23 Gümüş 2008, S. 62.

24 Vgl. Ministry of National Education 2009, S. 2-5. 
Gebiete an den Schulbetrieb bestehen in Form von Internaten, die Schüler ${ }^{25}$ aufnehmen, deren nächstgelegenes Dorf keine schulische Institution vorzuweisen hat und Busdiensten, die Kinder aus abgelegenen Gebieten unentgeltlich zur nächstgelegenen Dorfschule bringen. ${ }^{26}$ Diese Angebote sind jedoch nicht ausreichend, um die bezifferte Kluft zwischen städtischem Nordwesten und ländlichem Südosten der Türkei zu überwinden. Selbst wenn Kindern aus ländlichen Gebieten der Zugang zum Primarschulwesen gelingt, so sind sie trotz dessen benachteiligt, denn $»$ differences between village schools and city schools have persisted through to the current day ${ }^{27}$ : So sind in diesem Zusammenhang Unterschiede in Hinblick auf die Belegung der Klassenräume, das nummerische Lehrer-Schüler-Verhältnis und das zur Verfügung stehende Unterrichtsmaterial besonders eklatant. Die Zugangsrate von Schülern, die Dorfschulen besucht haben, zur Sekundarschule, ist außerdem signifikant geringer als die Raten bei städtischen Schulen. Religiöse weiterbildende Schulen, die eine İmam-Ausbildung bieten, werden hingegen vornehmlich von Absolventen der Dorfschulen besucht. ${ }^{28}$

\section{Geschlechtsspezifische Differenzen}

Aufgrund einer breit angelegten, von Weltbank und EU finanzierten Kampagne der UNICEF im Jahr 2003 konnte die Einschulungsrate von Mädchen in den letzten Jahren signifikant angehoben werden. Im Zusammenhang mit der Kampagne »Haydi Kızlar Okula! (» Mädchen, auf in die Schule! «) wurden die erschwerten Zugangsbedingungen von Mädchen zur Primarschulbildung untersucht und in Zeitungskampagnen sowie in der Zusammenarbeit mit Provinz-Behörden der Versuch unternommen, den sozialen Stellenwert von Schulbildung für Mädchen zu verbessern. ${ }^{29}$ Trotz der durch diese Kampagne erzielten Teilerfolge stellt der OECD-Bericht von 2007 zu Recht fest, »[t]he gender gap remains «. ${ }^{30}$ Auch hier lässt sich ein Gefälle zwischen nordwestlich gelegenen Metropolen und südöstlichen Provinzen beobachten. So gliedern sich die hohen Einschulungsraten im Primarschulwesen für das Schuljahr 2008/09, die in Istanbul bei 98,6 liegen, in

25 Sowohl hier als auch im Folgenden schließt Schüler weibliche und männliche Bildungsteilnehmer ein ebenso wie Lehrer, Absolventen etc. auch die weibliche Form impliziert.

Vgl. Gümüş 2008, S. 62-f., OECD 2007, S. 44.

27 Gümüş 2008, S. 62.

28 Vgl. ebd., S. 62. Imam-Hatip-Schulen nehmen Schülerinnen auf, auch wenn diese nicht İmam werden können. Die Ausbildung stellt in diesem Fall eine religiös geprägte Alternative zur allgemeinbildenden säkularen Sekundarschulbildung für Mädchen dar oder kann als Qualifikation zur Religionslehrerin genutzt werden. Vgl. Okçabol 2008, S. 94.

29 Vgl. UNICEF 2004.

30 OECD 2007, S. 46. 
98,9 bei Jungen und 98,1 Prozent bei Mädchen auf (Izmir: 99,2 Prozent mit Jungen 99,3, Mädchen 99,2 Prozent), während ländliche Gebiete mit niedrigen Zugangsraten eine größere Kluft vorweisen. So wurden zum Schuljahr 2008/o9 in der südöstlichen Provinz Van 91,7 Prozent der männlichen und lediglich 84,6 Prozent der weiblichen Bevölkerung eingeschult. ${ }^{31}$ Im Hinblick auf die ebenfalls geschlechterabhängige Abbrecherquote verstärkt sich diese Problematik, werden doch mehr Mädchen als Jungen noch vor Ende der Ableistung der Primarschulpflicht von der Schule genommen. ${ }^{32}$

\section{Qualitative Schwierigkeiten}

Laut der PISA-Studie von 2009 liegt die Türkei im Ländervergleich der Schülerleistungen in allen Kompetenzbereichen statistisch signifikant unter dem OECDDurchschnitt. ${ }^{33}$ Trotz verbreiteter Skepsis über die Aussagekraft von PISA-Ergebnissen lassen sich Schwierigkeiten im türkischen Schulsystem erkennen, die für das unterdurchschnittliche Abschneiden der Türkei im Ländervergleich mitverantwortlich sein dürften. Überfüllte Klassenzimmer und zu wenig pädagogisches Personal an öffentlichen Primarschuleinrichtungen wurden sowohl in der Studie des türkischen Lehrerverbandes Eğitim ve Bilim Emekçileri Sendikası (Ĕ̆itim-Sen) aus dem Jahr 2004 als auch im OECD-Bericht 2007 kritisiert $^{34}$ und sind, wenn auch mit leichten Verbesserungen, noch immer zu beobachten. Anhand der Zahlen der Bildungsstatistik 2008/o9 lässt sich bei permanenter Nutzung aller in öffentlichen Schulen zur Verfügung stehender Klassenräume eine durchschnittliche Belegung von 33 Schülern pro Klassenraum berechnen. Pro Lehrkraft sind außerdem im staatlichen Schulbetrieb durchschnittlich 24 Schüler $^{35}$ zu betreuen. Trotz des zentralisierten Lehrplans lässt sich daher feststellen, dass eklatante Differenzen in der Ausstattung von Schulen, der Qualifikation von Lehrenden und weiteren Divergenzen dazu führen, dass signifikante Unterschiede in der Durchführung des Lehrplanes an unterschiedlichen Schulen feststellbar sind. ${ }^{36}$

31 Vgl. Ministry of National Education 2009, S. 5.

32 Vgl. OECD 2007, S. $47 \mathrm{f}$.

33 Vgl. OECD 2010, S. 9.

34 Vgl. Gümüş 2008, S. 67 bzw. OECD 2007, S. 47.

35 Im Vergleich zu 18 im deutschen Primarschulbereich. Vgl. Ministry of National Education 2009, S. 53, Autorengruppe Bildungsberichterstattung 2010, S. 42. 


\section{Sozio-ökonomische Spaltung}

Qualitative Defizite lassen sich vor allem an frei zugänglichen, staatlichen Schulen feststellen. Die Lehrqualität wird nicht nur durch Unterschiede in Qualifikation und Fortbildung der Lehrkräfte beeinflusst, sondern leidet an staatlichen Schulen vor allem unter strukturellen Defiziten. Im Vergleich zur Klassenzimmerbelegung von 33 Schülern an staatlichen Schulen kommen in Privatschulen nur 16 Schüler auf einen Klassenraum. Auch im Schüler-Lehrende-Verhältnis lässt sich ein eklatanter Unterschied feststellen: Kommen im staatlichen Primarschulbereich 24 Schüler auf eine Lehrkraft, so sind es im privaten Primarschulbereich lediglich zehn Schüler pro Lehrkraft ${ }^{37}$ Diese Differenz weist auf eine starke Beeinflussung der Bildungschancen durch sozio-ökonomische Bedingungen hin, werden private Schulen in der Türkei doch aufgrund der Kostenpflicht vornehmlich von Kindern aus ökonomisch gutsituierten Familien besucht. Die Anzahl privater Schulen, die ein höheres Ansehen genießen als frei zugängliche Schulen und aufgrund höherer finanzieller Mittel bessere Betreuungsquoten und eine höhere Auswahl an Bildungsangeboten bieten können, steigt stetig an. Damit werden zwar staatliche Leistungsträger entlastet, doch die Auswirkungen des sozio-ökonomischen Hintergrundes beeinflusst zunehmend die Bildungschancen. ${ }^{38}$ Disparitäten bestehen folglich sowohl horizontal in Hinblick auf geografisch bedingte Unterschiede wie auch vertikal in Bezug auf sozio-ökonomische Hintergründe und das Geschlecht der Bildungsteilnehmenden.

Trotz wesentlicher Verbesserungen im Zugang zum türkischen Primarschulwesen lässt sich feststellen, dass eine gesteigerte Zugangsrate von nahezu 97 Prozent keinesfalls ein überzeugendes Argument ist, um eine fundierte Aussage über Zugangschancen von Bildungsteilnehmenden treffen zu können. Die eklatanten Unterschiede, die sich in den höchst divergenten Einschulungsraten von 84,6 Prozent der weiblichen Bevölkerung in der südöstlichen Provinz Van gegenüber der geschlechterübergreifenden hundertprozentigen Zugangsrate in wohlhabenden Istanbuler Teilorten spiegeln, weisen auf ein ernstzunehmendes Bildungsgefälle in der Türkei hin. Dies macht sich im Primarschulwesen entlang regionaler, sozio-ökonomischer und geschlechtlicher Unterschiede bemerkbar. Ob, wie vom Ministerium für Nationale Bildung angekündigt, bis 2013 eine aus einer sozioökonomisch benachteiligten Familie stammende Bildungsteilnehmerin im Hinterland der Provinz Van die gleichen Zugangschancen zur Primarschulbildung hat, die ein Junge aus einem wohlhabenden Haus in Istanbul genießt, bleibt zu er-

37 Gerundete Zahlen eigener Berechnung aus den Daten des Ministry of National Education 2009, S. 53.

38 Vgl. Gümüş 2008, S. 73. 
hoffen. Dies müsste jedoch mit umfassenden Reformen einhergehen, die an den soeben dargestellten Faktoren für die Kluft zwischen den Zugangschancen ansetzen müssten. Aufgrund der dargestellten problematischen Bedingungen im regulären staatlichen Schulsystem ist jedoch fraglich, ob die Kapazitäten für ein solches Vorhaben in den nächsten Jahren gegeben sein werden. Im Vergleich der Zugangsraten der Bildungsstatistiken von 2008/09 und 2010/11 lassen sich, wie bereits angesprochen, nur äußerst geringe Erfolge in der Verbesserung von $\mathrm{Zu}$ gangsraten im vorschulischen Bereich, der Anpassung der Zugangsraten von städtischen und ländlichen Gebieten sowie verbesserte Zugangschancen für weibliche Bildungsteilnehmer ablesen. Auch das im Folgenden noch zu erörternde im März 2012 verabschiedete Reformpaket lässt wenig Hoffnung, diese Differenzen überbrücken zu können.

\subsection{Sekundarschulwesen}

Der Besuch einer Bildungseinrichtung des Sekundarschulbereichs ist optional. Er folgt der Primarschulbildung und bietet eine mindestens vierjährige weiterführende Ausbildung für die Altersgruppe der 14- bis 18-Jährigen. Auch in diesem Bildungssektor wird der kostenfreie Zugang an staatlichen Schulen gewährleistet, während private Einrichtungen kostenpflichtig sind. ${ }^{39}$ Die Zugangsrate lag im Schuljahr 2008/o9 bei 58,5 Prozent der entsprechenden Altersgruppe und bleibt damit trotz steigender Zahlen in den letzten Jahren auch in diesem Bereich noch immer weit hinter den Raten anderer OECD-Staaten zurück. ${ }^{40}$

Der Sekundarschulbereich lässt sich aufgliedern in allgemeinbildende Schulen auf der einen sowie berufsbildende und technische Schulen auf der anderen Seite. Während die allgemeinbildenden Schulen ein vornehmlich theoretisches Lehrangebot vorzuweisen haben, um auf die Zugangsexamen zur Hochschule vorzubereiten, beinhalten berufsbildende und technische Schulen sowohl theoretische Schulbildung als auch praktische Lehre in Betrieben. ${ }^{41}$ Im Schuljahr 2008/09 besuchten 59 Prozent der sich in der Sekundarstufe befindenden Schüler allgemeinbildende Schulen und 41 Prozent berufsbildende und technische Schulen. ${ }^{42}$ Die Probleme, die bereits im Zusammenhang mit der Primarschulbildung geschildert wurden, bestehen im Bereich der Sekundarstufe zum Teil in noch höherem Maße. Die bereits im Mittel niedrige Zugangsrate minimiert sich in ländlichen Gebie-

39 Vgl. Ministry of National Education 2008, S. 5.

40 Vgl. ebd. 2009, S. 1 bzw. OECD 2007, S. 83.

41 Vgl. OECD 2007, S. 71-73, Okçabol 2008, S. 89-91.

42 Errechnet nach Daten aus Ministry of National Education 2009, S. 73. 
ten noch weiter, da nur eine sehr geringe Anzahl allgemeinbildender sowie berufsbildender und technischer Sekundarschulen außerhalb von Ballungsgebieten angesiedelt ist. ${ }^{43}$ Auch das Geschlechtergefälle verstärkt sich im Sekundarbereich: »The gender imbalance at the secondary level is more severe than at the primary school level. $\aleph^{44}$ Dies gilt vor allem für berufsbildende und technische Sekundarschulen. ${ }^{45}$ Das sozio-ökonomische Bildungsgefälle lässt sich auch hier an starken Unterschieden zwischen Zugangschancen und Unterschieden der Bildungsqualität an kostenpflichtigen privaten Schulen und frei zugänglichen Staatsschulen ablesen. ${ }^{46}$ Auch Lehrkraftmangel, mangelhafte Ausstattung und fehlende Einbindung der Lehreinrichtungen an Gremien der politischen Entscheidungsfindung lassen sich im Sekundar- wie im Primarschulbereich konstatieren.

\subsection{Hochschulwesen}

Während Vor-, Primar- und Sekundarschulwesen sowie die non-formale Bildung dem Ministerium für Nationale Bildung unterstellt sind, bildet das Hochschulwesen eine Ausnahme. »Higher education institutions are autonomous for purpose of teaching and research. «7 Diese Selbstständigkeit wird durch den Hochschulrat (Yükseköğretim Kurulu, YÖK) beaufsichtigt, der für Planung und Koordination des Hochschulwesens zuständig ist und dem die höheren Bildungseinrichtungen zu jährlicher Berichterstattung verpflichtet sind. ${ }^{48} \mathrm{Zu}$ den höheren Bildungseinrichtungen zählen Universitäten, Konservatorien, höhere berufsbildende Schulen sowie Forschungszentren, zu denen die Zugangsrate insgesamt 27,7 Prozent beträgt. ${ }^{49}$

Als problematisch und für die geringen Studienanfängerzahlen mitverantwortlich wird der zentrale Zugangstest zum Hochschulwesen betrachtet. Der landesweite kostenpflichtige Test wird mit dem Abschlusszeugnis der besuchten Sekundarschule verrechnet und bildet einen Mittelwert, dessen Höhe über die

43 Vgl. ebd., S. 75 .

44 OECD 2007, S. 79.

45 Vgl. Okçabol 2008, S. 95.

46 Vgl. ebd., S. 97.

47 Ministry of National Education 2008, S. 6.

48 Vgl. ebd. Eine absolute Autonomie des Hochschulrates besteht jedoch nicht, ist doch das Ministerium für Nationale Bildung für die Kontrolle der Einhaltung nationaler bildungspolitischer Richtlinien im Hochschulwesen zuständig. Vgl. Ministry of National Education June 2005 , S. 5.

49 Vgl. ebd. 2009, S. 1. Zum internationalen Vergleich: In Deutschland betrug die Quote 2009 43 Prozent, das OECD-Mittel liegt gar bei über 50 Prozent. Vgl. Autorengruppe Bildungsberichterstattung 2010, S. $121 \mathrm{f}$. 
Einstufung der Kandidaten bestimmt. Dieser wiederum muss über der Zugangsgrenze zur Hochschulbildung liegen und die Punktzahlen des jeweils gewünschten Faches und der universitären Einrichtung erreichen. ${ }^{50}$ Der selektive Zugang zu Sekundarschuleinrichtungen schlägt sich hier erneut nieder, wurden die Punktzahlen bis Dezember 2011 doch mit nach besuchter Schule und angestrebtem Studium aufgeschlüsselten Zahlen multipliziert, die für Schüler von İmam-HatipSchulen einen negativen Multiplikator darstellen konnten, während Schüler der angesehenen Anatolischen Sekundarschulen (Anadolu Lisesi) ${ }^{51}$ im gleichen Bereich einen positiven Multiplikator erhielten. ${ }^{52}$ Selbst nach der Reform, die zwar die Stellung berufsvorbereitender Gymnasien wie auch der Imam-Hatip-Schulen verbesserte, liegen die erzielten Punktezahlen von Schülern, die teure Privatschulen besucht haben, jedoch noch immer deutlich höher als die Punktzahlen von Schüler kostenloser und ländlicher schulischer Einrichtungen.

Die Zugangsberechtigung zu den Tests stellt zwar der Sekundarschulabschluss dar, aufgrund hoher Durchfallraten und fehlender Abstimmung des Sekundarschullehrplans mit den Zugangstests werden jedoch von 93 Prozent der zukünftigen Studienanfänger und -anfängerinnen private, kostenpflichtige Vorbereitungskurse in Anspruch genommen. ${ }^{53}$ Die hohe Selektivität des türkischen Bildungswesens setzt sich folglich auch in diesem Bereich fort und benachteiligt auch im Hochschulwesen Regionen, sozio-ökonomisch schwache Gruppierungen, Bildungsteilnehmerinnen und Kinder aus Familien mit religiösem Hintergrund, die ihren Kindern eine religiös geprägte Grundbildung zukommen lassen und ihnen dadurch den Zugang zu weiterbildenden Schulen erschweren.

\subsection{Sonderfälle}

Im formalen Bildungssektor gibt es für die dargestellten Bildungsbereiche neben dem regulären Schulbetrieb einige Sonderfälle, die hier nicht eingehend erörtert aber dennoch kurz skizziert werden sollen. Es handelt sich dabei um religiöse Bildungseinrichtungen, Bildung für Kinder und Jugendliche mit besonderen Bedürfnissen, Fernunterricht sowie ausländische und internationale Schulen.

50 Vgl. Varli 2008, S. 102.

51 Anatolische Sekundarschulen wurden als staatliche Alternative zu teuren privaten Bildungseinrichtungen mit Sprachenschwerpunkt eingerichtet. Der Unterricht wird nicht nur auf Türkisch, sondern auch in der entsprechenden Fremdsprache (Englisch, Deutsch oder Französisch) abgehalten. Schüler dieser Einrichtungen schneiden beim Zugangstest zur Hochschulbildung im Vergleich zu anderen staatlichen Gymnasien besonders erfolgreich ab.

52 Vgl. OECD 2007, S. 88.

53 Vgl. OECD 2007, S. 91, Okçabol 2008, S. 101. 


\section{Religiöse Bildungseinrichtungen}

Als bedeutendster Akteur im Bereich islamisch geprägter Bildung ist das Fethullah-Gülen-Netzwerk ${ }^{54}$ zu nennen, das zwar offiziell keine Schulen in der Türkei betreibt, dort allerdings seinen Ursprung hat und weltweit in über 80 Ländern schulische Einrichtungen leitet. Obwohl Gülen-Schulen offiziell aufgrund rechtlicher Schwierigkeiten, die mit dem Verbot religiöser Schulen in der Türkei einhergehen, nicht existieren, ist der Einfluss des Gülen-Netzwerkes auf zahlreiche schulische Einrichtungen in der Türkei offensichtlich und ein viel diskutiertes Phänomen in der türkischen Gesellschaft. ${ }^{55}$ Der Analyse Agais folgend, kann angenommen werden, dass seit den 1980er Jahren durch die Anhänger Gülens in der Türkei zirka 150 Privatschulen und 150 dersane ${ }^{56}$ sowie eine wesentlich höhere Anzahl an Wohnheimen für Schüler und Studenten entstanden sind. ${ }^{57}$

\section{Kinder und Jugendliche mit besonderen Bedürfnissen}

»Perhaps the most troubling aspect of the Turkish educational system concerns those children who require special education. ${ }^{{ }^{58}} \mathrm{Im}$ Rahmen der Bildungsreformen von 1997 wurde zwar gesetzlich festgelegt, dass für Menschen mit besonderen Bedürfnissen gleiche Chancen und Teilnahmebedingungen im Bildungswesen geschaffen werden müssten, jedoch scheint das türkische Bildungssystem von diesem Ziel noch weit entfernt. Verlässliche Daten sind aufgrund unterschiedlicher Definitionen besonderer Bedürfnisse und divergierender Zahlen sowie mangelhafter wissenschaftlicher Bearbeitung schwer zu erhalten. Laut Gümüş hatten im Schuljahr 2007/08 von zirka 250000 Kindern mit physischen und psychischen Benachteiligungen im Alter von fünf bis 14 Jahren lediglich etwa 80 ooo Kinder

54 Fethullah Gülen ist ein 1941 geborener türkischer Imam, der heute in den USA lebt und als Kopf eines internationalen islamischen Netzwerkes zu betrachten ist. Das Netzwerk versucht Islam und Modernität zu verbinden und beschäftigt sich vorrangig mit Bildung und interkulturellem Dialog, ist jedoch auch in Wirtschaft, Politik und Medien mit einer stetig wachsenden Zahl von Anhängern vertreten. Während dem Netzwerk von Kritikern vorgeworfen wird, den Bildungsschwerpunkt zum Zweck der Ausbildung einer islamischen Elite gewählt zu haben, äußert das Netzwerk selbst, es wolle die Möglichkeit bieten, islamische Spiritualität mit intellektueller Aufklärung zu verknüpfen und dem Islam den Weg in die Moderne zu ebnen. Vgl. beispielsweise Agai 2008.

55 Vgl. Yavuz, Esposito 2003.

56 Ein dersane (»Kurshaus «) ist eine private Bildungseinrichtung, die zusätzlich zum regulären Schulunterricht Nachhilfe anbietet und auf den zentralen Universitätsaufnahmetest in der Türkei vorbereitet.

57 Vgl. Agai 2008, S. 13.

58 Gümüş 2008, S. 63. 
Zugang zu schulischen Einrichtungen. ${ }^{59}$ Von diesen war der größte Teil in regulären Schulen untergebracht, deren Kapazitäten für integrativen Unterricht aufgrund der beschriebenen Problematik überfüllter Klassen und unzureichender Lehrendenzahl jedoch fragwürdig erscheint. ${ }^{60}$

\section{Fernunterricht}

Fernunterricht wird im Primar-, Sekundar- und Hochschulbereich angeboten. Aufgrund hoher Raten von Schulabbrechenden und Menschen, die lediglich fünf Jahre die Grundschule besucht haben, wird das Angebot des Selbststudiums meist als weiterbildendes Angebot von Erwachsenen genutzt, die ihre Bildungslaufbahn abgebrochen haben oder aufgrund geografisch bedingter Schwierigkeiten keinen Zugang zur Schulbildung hatten. Vor allem weibliche Bildungsteilnehmer mit Kindern sollen von den Angeboten profitieren können. Außerdem bietet das Programm Bildungschancen für im Ausland lebende türkische Staatsbürger und ein wachsendes Angebot des universitären Fernstudiums. ${ }^{61}$ Mit Blick auf die Umsetzung lässt sich im Fernunterricht-Angebot des Ministeriums für Nationale Bildung für die Primar- und Sekundarstufe feststellen, dass die Zahl der sich jährlich neu einschreibenden Schüler zwar stetig steigt und sich aufgrund computergestützter Programme noch einmal vervielfacht hat, die Zahl der jährlichen Abschlüsse jedoch diesem Trend nicht folgen kann. ${ }^{62}$

\section{Internationale und ausländische Schulen}

Neben staatlichen und privaten türkischen Schulen werden vor allem in den Metropolen Istanbul und Ankara auch internationale und ausländische Schulen betrieben. Diese sind entweder an Botschaften angegliedert und damit dem Lehrplan des jeweiligen Botschaftslandes verpflichtet oder es handelt sich um private Institutionen, die zumeist internationale Schulen betreiben. Als Beispiel sei die British International School Istanbul genannt, eine nach britischem Lehrplan unterrichtende Privatschule, die Vor-, Primar- und Sekundarbereich mit erweitertem Sport- und Freizeitangebot anbietet. Aufgrund der Gebühren von zirka

59 Vgl. ebd.

60 Vgl. OECD 2007, S. 119-123, Sari 2000.

61 Vgl. Ministry of National Education 2007, S. 14

62 Während die Neuzugänge im Sekundarschulbereich von 17500 im Schuljahr 1993/94 auf 231800 2011/12 stetig angestiegen sind, unterliegen die Abschlussraten extremen Schwankungen und liegen 2011/12 bei 32 800, also bei zirka 14 Prozent der Neuzugänge. Vgl. Açık Öğretim Lisesi 2012. 
25000 US-Dollar für die ersten fünf Grundschuljahre dürfte diese jedoch nur einem extrem kleinen Kreis von Interessierten zugänglich sein.

\section{Das türkische Bildungssystem im Spannungsfeld zwischen divergierendem nationalen und inter- nationalen Einfluss: die Bildungsreform 2012}

Im türkischen Bildungssystem lässt sich folglich nicht nur eine flächendeckende qualitative Problematik feststellen, sondern es müssen auch ein gravierendes Bildungsgefälle zwischen elitären und öffentlichen Einrichtungen sowie erschwerte Zugangsbedingungen für die Bevölkerung aus ländlichen Gebieten, weibliche Bildungsteilnehmer und für Kinder und Jugendliche mit besonderen Bedürfnissen konstatiert werden. Der im Zusammenhang mit dem Zuständigkeitsbereich des Ministeriums für Nationale Bildung bereits skizzierte zentralisierte Aufbau des Bildungssystems " continues to eschew participation and pluralism in favor of authoritarianism and conservatism. $\aleph^{63}$ Trotz wiederholter Forderungen wurde auch im Laufe der Reformen von 1997 keine maßgeblichen Veränderungen an Aufbau und Zuständigkeit des Ministeriums durchgesetzt, »because of central bureaucracy's resistance to change. « ${ }^{64} \mathrm{Nohl}$ fasst die anhaltende Monopolstellung des Ministeriums passend zusammen: » The Turkish education system is centralist. All major decisions are taken at the Ministry of National Education [...] and their implementation is controlled from there. ${ }^{65}$ Die Implementierung einer einheitlichen Bildungslandschaft hat dies jedoch nicht zur Folge. Stattdessen spiegeln sich in den zahlreichen Schwierigkeiten des Bildungssystems Auseinandersetzungen zwischen divergierenden politischen Ausrichtungen wie Säkularismus, Islamismus, kemalistischem Nationalismus, Konservatismus und Liberalismus wieder.

Die Vermittlung nationaler Werte im Schulunterricht wird aufgrund entsprechender Vorgaben durch das Ministerium für Nationale Bildung mit Ritualen wie dem täglichen Aufsagen des Schuleids sowie dem Hissen der türkischen Flagge und Singen der Nationalhymne an jedem Montag und Freitag schon seit Jahrzehnten landesweit durchgesetzt. ${ }^{66}$ Eine den pluralistischen Ansprüchen verschie-

63 Gümüş 2008, S. 78.

64 Dulger 2004, S. 18.

65 Nohl 2008, S. 43. Die zentralistische Organisation des Bildungswesens schlägt sich in einem hohen Maß an Gleichförmigkeit nieder, die sowohl äußerlich in der Form von einheitlichen Schulgebäuden und Schuluniformen, als auch inhaltlich in Form einheitlicher Lehrpläne und Schulbücher erkenntlich ist. Vgl. Karakaşoğlu-Aydin 2002, S. 561.

66 Zur Vermittlung nationaler Werte im Schulunterricht vgl. Gümüş 2008, S. 68, Altınay 2004, S. $117 \mathrm{ff}$. 
dener gesellschaftlicher Gruppierungen entsprechende, sich einzelnen Ideologien entziehende Ordnung des Bildungswesens lässt sich hingegen bisher nicht erkennen. Aus diesem Grund schaffen sich religiöse Gruppierungen mit İmamHatip-Schulen ebenso wie die elitären Kreise mit privaten Einrichtungen höheren Bildungsniveaus einen eigenen Bildungssektor. Das zentralistische und den kemalistischen Werten verpflichtete Bildungssystem der Türkei erreicht folglich nicht, wofür es sich seit der Gründung der Republik verpflichtet hat: die Vereinheitlichung des nationalen Bildungsstandards auf einem ausreichend hohen Niveau.

Unter Druck gerät das System außerdem durch den wachsenden Einfluss internationaler Akteure. OECD, Weltbank, Europäische Kommission, UNICEF und UNESCO seien hier nur als einige wenige genannt, die mittlerweile immensen Einfluss auf das türkische Bildungssystem ausüben. Kampagnen, die von internationalen Organisationen in der Türkei durchgeführt werden, verstärken die Aufmerksamkeit der internationalen Gemeinschaft. Die externe Finanzierung dieser Kampagnen durch Weltbank und Europäische Kommission verpflichten den türkischen Staat außerdem ganz direkt zu Berichterstattung und Anpassung der bildungspolitischen Richtlinien an die des jeweiligen Geldgebers. Damit befindet sich das türkische Bildungssystem unter dem Einfluss verschiedenster Akteure und muss sowohl Bedürfnissen von außen gerecht werden als auch der inhärenten Spannung, die in der Türkei besteht zwischen "nationalism and positivism, nationalism and religion, skepticism about the West and positivism, and a strong central government and democratic equality. « ${ }^{67}$

Als aktuelles Spiegelbild der inneren politischen Auseinandersetzungen um das Bildungssystem lässt sich die im März 2012 verabschiedete Bildungsreform anführen. Die Reform, die unter dem Titel » $4+4+4$ " publik geworden ist, sieht vor, die obligatorische Schulzeit von bisher acht auf nunmehr zwölf Jahre anzuheben. Diese Verlängerung der Schulpflicht scheint auf den ersten Blick eine Anpassung an internationale Bildungsstandards darzustellen. Die innenpolitischen Auseinandersetzungen, die mit der Einführung der Reform einhergingen, zeichnen jedoch ein anderes Bild. Die Reform wurde, noch bevor diese öffentlich bekannt und diskutiert worden wäre, am 11. März 2012 unter gewaltsamem Ausschluss der Opposition von der Regierungspartei AKP verabschiedet. ${ }^{68}$ Die Verabschiedung führte nicht nur zu heftigen Wortgefechten zwischen AKP und Oppositionsparteien, sondern rief auch Massendemonstrationen in den Metropolen hervor.

67 Gümüş 2008, S. 78 f.

68 Die Bilder von Abgeordneten der Opposition, die versucht hatten, sich Zugang zum Sitzungssaal zu verschaffen und sich im Zuge dessen mit Sicherheitskräften und AKP-Abgeordneten Handgefechte lieferten, bestimmten landesweit die Presse. Vgl. »Turkish deputies injured in fighting as education bill passes«. Hürriyet Daily News, 11. März 2012. 
Grund für die erbitterte Auseinandersetzung ist die Angst Oppositioneller und breiter Teile der Bevölkerung, die AKP wolle mit der Reform den Weg ebnen für den Zugang zu religiösen Bildungseinrichtungen im frühen Kindesalter ( $a b$ neun Jahren) und schaffe außerdem die Möglichkeit, Mädchen in Zukunft leichter dem Bildungswesen zu entziehen. Diese Angst scheint berechtigt, nicht nur aufgrund der zweifelhaften Verabschiedung des Gesetzes, sondern auch mit Blick auf dessen Inhalt.

So wird die zwölfjährige Schulpflicht in Zukunft in drei Abschnitten zu jeweils vier Jahren abzuleisten sein, wie bereits der Name »4+4+4 « impliziert. Nach den ersten vier Schuljahren (Fünf- bis Achtjährige) steht es den Eltern jedoch frei, ihr Kind auf eine religiöse Schule zu schicken. Außerdem muss der Anschluss an die nächste Schulstufe nicht sofort erfolgen, sondern es wird Raum für Lücken zwischen den jeweiligen vier Jahren gelassen und die letzten vier Jahre können als Fernstudium geleistet werden. Betrachtet man die oben bereits erwähnten extrem niedrigen Abschlussraten bei Fernstudien der Sekundarstufe, so stellt diese Option eine Farce dar. Die Opposition argumentiert daher, dass das Gesetz eingeführt worden sei, um einen früheren Einstieg in die religiösen Imam-HatipSchulen zu ermöglichen, in die bisher erst nach der achtjährigen Schulpflicht im Alter von zirka 14 Jahren eingetreten werden konnte. Auch die Gefahr, dass Mädchen nach den ersten vier Jahren nicht in eine weiterführende Schule geschickt werden, lässt sich nicht von der Hand weisen, besteht doch noch immer vor allem im Südosten der Türkei ein eklatantes geschlechterspezifisches Bildungsgefälle. Die Regierung argumentiert in diesem Zusammenhang, die zwölf Jahre müssten obligatorisch abgeleistet werden und dies würde notfalls von staatlicher Stelle überwacht und durchgesetzt werden. Mit Blick auf die Schwierigkeiten, die selbst bei der Durchsetzung eines einzigen Blocks von acht Jahren noch immer bestehen, erscheint dieses Argument jedoch wenig überzeugend.

Die Spannung zwischen religiöser Regierungspartei und säkularer Opposition lässt sich an diesem Beispiel deutlich erkennen und wird von Bemerkungen von Ministerpräsident Recep Tayyip Erdoğan keinesfalls entschärft. ${ }^{69}$ Wie sich die Bildungsreform nach ihrer Umsetzung tatsächlich auswirken wird, bleibt abzuwarten. Die mit ihr einhergehenden Kämpfe um Einfluss auf das Bildungssystem aus verschiedenen politischen und gesellschaftlichen Gruppierungen sind jedoch of-

69 Dieser äußert in Bezug auf die mit der Reform einhergehenden neuen Möglichkeit, in allen schulischen Einrichtungen Korankurse als Wahlfach zur Verfügung zu stellen: »What is more beautiful than learning the Quran? Today, through our government, this nation has attained the liberty to learn its religion to the full. « (»Education reform will erase traces of Feb 28: Turkish PM «. Hürriyet Daily News, 31. März 2012; vgl. außerdem zur Bildungsreform Finkel 2012.) 
fensichtlich. Welchen Einfluss Gruppierungen, die reformpädagogische Ansätze umzusetzen versuchen, auf das Bildungswesen ausüben, steht im Zentrum der abschließenden Fragestellungen. Dabei wird argumentiert, dass alternative Bildungsstrategien und der verstärkte Einfluss zivilgesellschaftlicher Kräfte zu einer Dezentralisierung und Pluralisierung der türkischen Bildungslandschaft führen könnten, die als notwendige Wege für eine nachhaltige Modernisierung und Demokratisierung der Türkei zu bewerten sind.

\section{International diffundierende alternative Bildungs- strategien: zivilgesellschaftlicher Einfluss}

Neben staatlichen Initiativen, die das reformbedürftige Bildungssystem der Türkei zu modifizieren suchen, und internationalen Akteuren, die Einfluss auf Reformen ausüben, greifen seit einigen Jahren auch zivilgesellschaftliche Akteure verstärkt in die Bildungslandschaft ein. Diese arbeiten daran, alternative, international diffundierende Bildungsstrategien (wie etwa Montessori, Waldorf, Summerhill, Reggio usw.) auch in der Türkei umzusetzen. Alternative Bildungsstrategien im Sinne von pädagogischen Konzepten und Lehrplänen, die vom staatlichen Ansatz des Ministeriums für Nationale Bildung abweichen, sind aufgrund des zentralisiert organisierten und homogenisierten Systems in der Türkei nur in äußerst begrenztem Ausmaß vorhanden. Bisher konnten in der Türkei keine Schulen eröffnet werden, die offiziell vom Lehrplan und dem pädagogischen Konzept des Ministeriums abweichen. Die Schwierigkeiten, denen sich alternative Bildungsbewegungen ausgesetzt sehen, sind im Licht historischer Prozesse zu sehen, die für eine Auflösung des starren Bildungskonzeptes bisher nicht förderlich gewesen zu sein scheinen.

Im internationalen Vergleich ist die fehlende bzw. schwach ausgeprägte Umsetzung der Konzepte bemerkenswert, weisen doch selbst die weniger an europäisch-westlichem Bildungssystem orientierten Staaten wie Pakistan, Ecuador und Saudi Arabien etwa weiterführende Montessori-Schulen auf. Die Implementation alternativer Bildungskonzepte in der Türkei hingegen beschränkt sich bisher vornehmlich auf den vorschulischen Bereich und lässt sich in den Metropolen Istanbul, Ankara und Izmir verorten. Als Bildungskonzept wird dabei meist die Montessori-Pädagogik gewählt, die sich aufgrund ideologischer Offenheit mit den Vorgaben des Ministeriums für Nationale Bildung in Übereinstimmung bringen lässt. Als einzige reformpädagogische Bildungseinrichtungen in der Türkei lassen sich Montessori-Kindergärten in Istanbul, Ankara und Izmir nennen, die trotz reformpädagogischem Konzept weiterhin den Vorgaben des Ministeriums verpflichtet sind. 
Neben der geringen Anzahl umgesetzter reformpädagogischer Projekte existieren jedoch zahlreiche Initiativen, die neuartige Kindergarten- und Schulprojekte vorantreiben. So besteht eine Arbeitsgruppe zur Eröffnung einer »demokratischen Schule « mit dem Namen »Eine andere Schule ist möglich " (»Başka Bir Okul Mümkün «, BBOM), die in mehreren Metropolen ab 2013 Schulen betreiben werden, eine Waldorfinitiative und neben einigen Montessori-Vorschulen auch zusätzliche Montessori-Klassen an Primar- und Sekundarschulen sowie Initiativen zur Eröffnung von weiterführenden Montessori-Schulen. Die zivilgesellschaftliche Bewegung, die in allen Metropolen der Türkei zu finden ist und unterschiedliche international diffundierende Bildungsansätze nutzt, um zusätzliche schulische und vorschulische Angebote für ihre Kinder zu schaffen, bildet einen wachsenden und zunehmend ernstzunehmenden Einflussfaktor auf das türkische Bildungssystem. Die Bewegung setzt sich vornehmlich aus der jungen Elternschaft akademischer Kreise zusammen, die im Ausland studiert oder gearbeitet hat und nach ihrer Rückkehr in den akademischen Dienst eingetreten ist. Aufgrund ihrer Erfahrungen mit fremden Bildungssystemen fordern diese Eltern eine erweiterte Bildungschance für ihre eigenen Kinder. Die selbstzugeschriebene Zugehörigkeit zur wachsenden Mittelschicht in der Türkei stellt sie dabei vor das Problem, zwischen kostenfreien staatlichen Schulen, die aufgrund der dargestellten Mängel als unzureichend betrachtet werden, und teuren Privatschulen in oftmals ausländischer Trägerschaft wählen zu müssen. Um diesem Dilemma zu entkommen und die Defizite des Bildungssystems zu überbrücken, entscheiden sich immer mehr junge gebildete Eltern in der Türkei, den Ausweg aus dem Bildungsdilemma zu beschreiten, ohne auf die Umsetzung von Reformen seitens des Staates zu warten.

Besonders im Bereich vorschulischer Bildung kommt dies staatlichen Akteuren nicht ungelegen, tragen die privat betriebenen Einrichtungen doch zur im Wahlkampf versprochenen Verbesserung der Zugangsraten zu vorschulischer Bildung bei, ohne zusätzliche Kosten zu verursachen. Auch im primar- und sekundarschulischen Bereich werden derzeit Bestrebungen zur Umsetzung solcher Projekte unternommen, die von den Umwälzungen im Bildungssystem durch die "4+4+4«-Reformen, wie kritisierbar diese auch sein mögen, profitieren werden, da die Reform einen früheren Zugang nicht nur zu religiösen, sondern auch zu alternativpädagogischen Schulen schaffen wird. Ob alternativpädagogische Projekte für junge akademische Eltern in der Türkei einen Ausweg aus dem defizitären Bildungssystem bieten können und wie erfolgreich sich die Bewegung auch mit Blick auf die allgemeine Bildungsreform gestalten wird, bedarf der weiteren Forschung. 


\section{Quellen- und Literaturverzeichnis}

Açık Öğretim Lisesi 2012. Açık Öğretim Lisesine Ait Sayısal Veriler. http://www.aol.meb.gov. tr/sayfa_goster.asp? ID=126. Zugegriffen: 22. August 2012.

Agai, B. 2008. Zwischen Netzwerk und Diskurs. Das Bildungsnetzwerk um Fethullah Gülen (geb. 1938): die flexible Umsetzung modernen islamischen Gedankenguts. Schenefeld: EB-Verlag.

Altınay, A. G. 2004. The Myth of the Military Nation. Militarism, Gender, and Education in Turkey. New York: Palgrave Macmillan.

Autorengruppe Bildungsberichterstattung (Hrsg.) 2010. Bildung in Deutschland 2010. Ein indikatorengestützter Bericht mit einer Analyse zu Perspektiven des Bildungswesens im demografischen Wandel. Bielefeld: W. Bertelsmann Verlag.

Deutsches Statistisches Bundesamt 2010. Bevölkerung und Erwerbstätigkeit. Bevölkerung mit Migrationshintergrund. Ergebnisse des Mikrozensus 2009. Wiesbaden.

Döbert, H. (Hg.) 2002. Die Schulsysteme Europas. Baltmannsweiler: Schneider-Verlag. Hohengehren.

Dulger, İ. 2004. Turkey: Rapid Coverage for Compulsory Education. The 1997 Basic Education Program. Shanghai: The International Bank for Reconstruction and Development/The World Bank.

»Education reform will erase traces of Feb 28: Turkish PM «. Hürriyet Daily News, 31. März 2012. http://www.hurriyetdailynews.com/education-reform-will-erase-traces-offeb-28-turkish-pm.aspx?pageID=238\&nid=17350\&NewsCatID=338. Zugegriffen: 17. April 2012.

Finkel, A. 2012. What's $4+4+4$ ? International Herald Tribune, 23. März 2012. http://latitude.blogs.nytimes.com/2012/03/23/turkeys-education-reform-bill-is-about-playingpolitics-with-pedagogy/. Zugegriffen: 17. April 2012.

Gökdemir, F. 200o. Bildungssystem und Phänomene interkultureller Sozialisation in der türkischen Gesellschaft. Dissertation. Universität Paderborn.

Gümüş, A. 2008. Turkish Primary Education. Structure and Problems. In Education in Turkey, hrsg. A.-M. Nohl, A. Akkoyunlu-Wigley, S. Wigley, 49-81. Münster: Waxmann.

İlhan, E. 2012. Educating Turks. Mishandling Reform Could be the Undoing of the Justice and Development Party. Cairo Review of Global Affairs, Special Report: Turkey 4: 84-95.

İnal, K. 2011. AKP, Bildungsreform und Anpassung an den globalen Wandel. In Bildung und sozialer Wandel in der Türkei - Historische und aktuelle Aspekte, hrsg. A.-M. Nohl, B. Pusch, 45-78. Würzburg: Ergon.

İstanbul İl Milli Eğitim Müdürlügü 2012. 2011-2012 İstatistik Verileri. http://istanbul. meb.gov.tr/upload/bolumler/strateji/istatistik/istatistik_ist2012.pdf, Zugegriffen: 28. 08. 2012.

Kaplan, S. 2006. The pedagogical state. Education and the politics of national culture in post1980 Turkey. Stanford (CA): Stanford University Press.

Karakaşoğlu-Aydin, Y. 2002. Türkei. In Die Schulsysteme Europas, hrsg. H. Döbert, 558-574. Baltmannsweiler: Schneider-Verlag Hohengehren.

Ministry of National Education 2005. National Education Policy Review. Background Report. Ankara. 
Ministry of National Education 2008. The Development of Education. National Report of Turkey for the International Conference on Education, Genf, 25.-28. November 2008, Ankara. http://www.ibe.unesco.org/National_Reports/ICE_20o8/turkey_NRo8.pdf. Zugegriffen: 17. April 2012.

Ministry of National Education 2009. Milli Eğitim İstatistikleri - Örgün Eğitim. Ankara. http://sgb.meb.gov.tr/istatistik/meb_istatistikleri_orgun_egitim_2008_2009.pdf. Zugegriffen: 17. April 2012.

Ministry of National Education 2011. Milli Eğitim İstatistikleri - Örgün Eğitim. Ankara. http://sgb.meb.gov.tr/istatistik/meb_istatistikleri_orgun_egitim_2010_2011.pdf. Zugegriffen: 17. April 2012.

Nohl, A.-M., Akkoyunlu-Wigley, A., Wigley, S. (Hrsg.) 2008. Education in Turkey. Münster: Waxmann.

Nohl, A.-M., Pusch, B. (Hrsg.) 2011. Bildung und sozialer Wandel in der Türkei - Historische und aktuelle Aspekte. Würzburg: Ergon.

OECD 2007. Basic Education in Turkey. Reviews of National Policies for Education. http:// www.sourceoecd.org/9264030204. Zugegriffen: 17. April 2012.

OECD 2010. PISA 2009. Ergebnisse. Zusammenfassung. http://www.oecd.org/dataoecd/ 34/19/46619755.pdf. Zugegriffen: 17. April 2012.

Okçabol, R. 2008. Secondary Education in Turkey. In Education in Turkey, hrsg. A.-M. Nohl, A. Akkoyunlu-Wigley, S. Wigley, 83-105. Münster: Waxmann.

Sari, H. 200o. Development of Special Education Provision in Turkey. From the Inclusive Perspective. Paper presented at the International Special Education Congress Manchester, 24.-28. Juli 2000. http://www.isec20oo.org.uk/abstracts/papers_s/sari_1. htm. Zugegriffen: 17. April 2012.

Schriewer, J., Holmes, B. (Hrsg.) 2003. Theories and Methods in Comparative Education. Frankfurt am Main: Peter Lang.

»Turkish deputies injured in fighting as education bill passes «. Hürriyet Daily News, 11. 03. 2012. http://www.hurriyetdailynews.com/turkish-deputies-injured-in-fighting-as-education-bill-passes-.aspx?pageID=238\&nid=15746. Zugegriffen: 17. April 2012.

UNICEF 2004. Haydi Kizlar Okula! The Girl's Education Campaign in Turkey. http://www. unicef.org/turkey/pdf/ge6a.pdf. Zugegriffen: 17. April 2012.

UNICEF 2010. Turkey - Background. http://www.unicef.org/infobycountry/Turkey.html. Zugegriffen: 17. April 2012.

Varli, Z. 2008. The Structure of the Turkish Educational System. Practice and Theory in Systems of Education 3 (2): 95-103.

Yavuz, M.H., Esposito, J. L. 2003. Turkish Islam and the secular state. The Gülen movement. Syracuse, N.Y: Syracuse University Press.

Open Access. This chapter is distributed under the terms of the Creative Commons Attribution Noncommercial License, which permits any noncommercial use, distribution, and reproduction in any medium, provided the original author(s) and source are credited. 